\section{No slip-up}

\section{Crombie}

The Journal of Essential Oil Research. Editor-in-chief Brian M. Lawrence, Allured Publishing Corporation, 214 West Willow, PO Box 318, Wheaton, IIIinois 60189, USA. 6/yr. US and Canada $\$ 150$, elsewhere $\$ 170$.

THE term 'essential oil' does not convey anything very precise to many people. Indeed, it is a broad term, signifying the mixture of volatile organic compounds that produces the essential odour or taste of the vegetable substance from

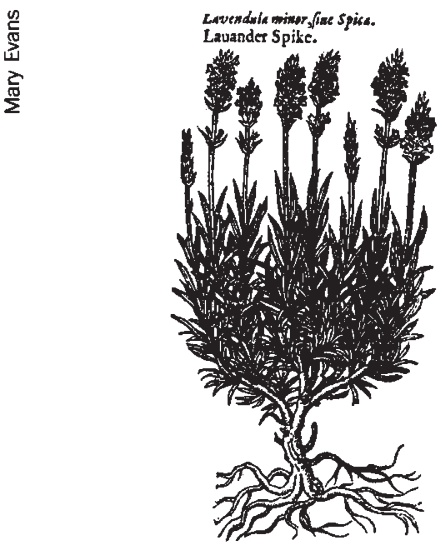

which it is derived. Evocative names like rose, eucalyptus, lavender, lemongrass, peppermint, patchouli, bergamot, vetiver and ylang-ylang are typical. Generally the compounds are produced by steam distillation or solvent extraction of suitable plant material, although occasionally more subtle and expensive means are employed to retain a delicate fragrance, as in the enfleurage process for jasmine products. Here the odorous compounds are collected by absorption into odourless lard from batches of flowers spread repeatedly onto glass plates smeared with the lard.

Commercially, essential oils are of particular importance in the perfumery and food-flavour industries; some are bulk commodities, some are extremely rare and expensive. Coming as they do from all parts of the globe, their supply depends on climatic and political circumstances, and, especially in the case of rare oils, price and supply can fluctuate and lead to adulteration with cheaper materials. Consequently, this area of analytical science of essential oils is an important, if specialized, one.

Organic chemists, too, have always been fascinated by essential oils; indeed, they have played an important role in the development of chemistry. The great German chemist Otto Wallach undertook prolonged investigation of essential oils from around 1880 onwards. This was a time when scientific knowledge and techniques were ill suited to dealing with such complex, often liquid, mixtures, yet by his labours he laid the foundation of terpene chemistry as we know it. Today life is much easier for the essential-oil chemist. Mass spectrometry coupled to separating equipment such as a gasliquid chromatograph, a high-performmance liquid chromatograph, or a second mass spectrometer, makes short work of the extremely complex mixtures if the components are already known and represented in the mass-spectrometry file. Even if new, much progress can be made on such structures. With powerful qualitative and quantitative tools such as these, adulteration is comparatively easy to detect.

The aims of the new journal, however, are much wider than this. It sets out to be

\section{Nouvelle cuisine}

\section{Gordon C. Cheeseman}

Trends in Food Science and Technology. Editor John O'Brien. Elsevier. 12/yr. $£ 239$ (institutional); UK £59, US and Canada \$92, elsewhere $£ 63$ (personal).

THIS new journal in the Trends series was launched by Elsevier in July 1990. A principal objective of the journal is to monitor and assimilate the significant innovations from a wide range of research areas, in order to stimulate research in the food sector.

Food science and technology is multidisciplinary, and a new journal in the field will always have the problem of maintaining the interest of specialists working in many fields. These fields include, for example, engineering, physics, chemistry, microbiology, nutrition and also involve the vertical spectrum of interests from basic food production, through processing, to diet and health.

The format used is of the magazine style and is ambitious in the number of sections proposed. These comprise features, reviews, viewpoints, letters, conference reports, book reviews, software reviews, technical advice and details of forthcoming events. But not all of these sections are included in each issue.

The main part of the journal contains review articles. The policy of keeping the reviews short and readable together with that of having a mix of topics in each issue is particularly valuable, as nonspecialists will be interested and kept informed. Specialists will find more extensive reviews published elsewhere; however, one advantage of a journal of this type is that the short publication time enables up-to-date research to be covered. The reviews appearing during devoted to all aspects of pure and applied essential-oil studies as summarized in the following categories: agriculture and horti culture, analytical chemistry, biological activity, biotechnology, chemical composition, chemical synthesis, chemosystematics, microbiology, plant biochemistry and biosynthesis and toxicology. Time will tell whether all these areas will be strongly represented in the journal. It accepts reviews, research communications, research reports, research letters and research notes, all in English. The journal is well produced on high quality paper, but personally I would have liked a larger format.

L. Crombie is in the Department of Chemistry, University of Nottingham, Nottingham NG7 2RD, UK.

the first year of publication have, in the main, been well written and usually sufficiently critical to enable the reader to obtain an appreciation of the likely impact and progress of the area of science covered. This aspect is important as the lag period between scientific advances and practical applications can be extensive if the important implications are not widely understood within the industry. There are usually three reviews per issue, and topics covered during the past year have included the detection of irradiated foodstuffs, molecular dynamics of food proteins, and electrical detection of food-borne microorganisms.

Interesting and useful inclusions are the viewpoints - occasional articles by leading experts in which more contentious areas are discussed. These have included articles on toxicity of diseaseresistant plants and water activity - a credible measure of food safety and quality. Such articles succinctly draw attention to specific problem areas for the food scientist, giving useful criticism of the body of published papers and highlighting problems with existing concepts.

The conference reports, instrumental and technical advice applicable to food research, book reviews and letters, all contribute to the magazine style of the journal.

Increased public awareness of diet and health relationships, changes in both life styles and retailing and manufacturing of food, has meant that a great deal of attention is now given to food research both in the public and private sectors. Interactive journals, such as Trends in Food Science and Technology, will play an important part in supporting the diverse and rapidly advancing area of food science.

Gordon C. Cheeseman is in the Department of Food Science and Technology, University of Reading, Reading, RG6 2AP, UK. 